\title{
Universal dependences between turbulent and mean flow parameters in stably and neutrally stratified Planetary Boundary Layers
}

\author{
I. N. Esau ${ }^{1,2}$ and S. S. Zilitinkevich ${ }^{1,2,3}$ \\ ${ }^{1}$ Nansen Environmental and Remote Sensing Centre, Thormohlensgate 47, 5006 Bergen, Norway \\ ${ }^{2}$ Bjerknes Centre for Climate Research, University of Bergen, Bergen, Norway \\ ${ }^{3}$ Division of Atmospheric Sciences, Department of Physical Sciences, University of Helsinki, Finland \\ Received: 26 July 2005 - Revised: 7 February 2006 - Accepted: 7 February 2006 - Published: 8 May 2006 \\ Part of Special Issue "Turbulent transport in geosciences"
}

\begin{abstract}
We consider the resistance law for the planetary boundary layer (PBL) from the point of view of the similarity theory. In other words, we select the set of the PBL governing parameters and search for an optimal way to express through these parameters the geostrophic drag coefficient $C_{g}=u_{*} / U_{g}$ and the cross isobaric angle $\alpha$ (where $u_{*}$ is the friction velocity and $U_{g}$ is the geostrophic wind speed). By this example, we demonstrate how to determine the "parameter space" in the most convenient way, so that make independent the dimensionless numbers representing co-ordinates in the parameter space, and to avoid (or at least minimise) artificial self-correlations caused by the appearance of the same factors (such as $u_{*}$ ) in the examined dimensionless combinations (e.g. in $C_{g}=u_{*} / U_{g}$ ) and in dimensionless numbers composed of the governing parameters. We also discuss the "completeness" of the parameter space from the point of view of large-eddy simulation (LES) modeller creating a database for a specific physical problem. As recognised recently, very large scatter of data in prior empirical dependencies of $C_{g}$ and $\alpha$ on the surface Rossby number $\mathrm{Ro}=U_{g}\left|f z_{0}\right|^{-1}$ (where $z_{0}$ is the roughness length) and the stratification characterised by $\mu$ was to a large extent caused by incompactness of the set of the governing parameters. The most important parameter overlooked in the traditional approach is the typical value of the Brunt-Väisälä frequency $N$ in the free atmosphere (immediately above the PBL), which involves, besides Ro and $\mu$, one more dimensionless number: $\mu_{N}=N /|f|$. Accordingly, we consider $C_{g}$ and $\alpha$ as dependent on the three (rather then two) basic dimensionless numbers (including $\mu_{N}$ ) using LES database DATABASE64. By these means we determine the form of the dependencies
\end{abstract}

Correspondence to: I. N. Esau

(igore@nersc.no) under consideration in the part of the parameter space representing typical atmospheric PBLs, and provide analytical expressions for $C_{g}$ and $\alpha$.

\section{Introduction}

Recently the resistance and heat transfer laws for the neutral and stable planetary boundary layers (PBLs) have been discusses and generalised by Zilitinkevich and Esau (2005). The reader can find an overview and the background physics in that paper. In the present paper we focus on the two parameters of particular practical interest: the geostrophic drag coefficient $C_{g}=u_{*} / U_{g}=u_{*} f \rho / \nabla P$ and the surface crossisobaric angle $\alpha$. Traditionally (Hess and Garratt, 2002) it was assumed the $C_{g}$ and $\alpha$ are controlled by the following set of external ${ }^{1}$ parameters: the surface roughness length $z_{0}$, the Coriolis parameter $f$, the geostrophic wind speed $U_{g}$ (or alternatively the mean horizontal pressure gradient $\nabla P$, because $U_{g}=\nabla P /(\rho f)$, where $\rho$ is the fluid density), and the potential temperature flux at the surface $F_{\theta}$.

The resistance law for the stratified PBLs was derived in the late sixties by Zilitinkevich et al. (1967) and Zilitinkevich (1989) through asymptotic matching of near-surface wind

\footnotetext{
${ }^{1}$ We consider $F_{\theta}$ as external parameter. In the present paper this is justified because we use LES data generated with given values of $F_{\theta}$. However, in the heat transfer problem $F_{\theta}$ is an unknown that should be determined through an alternative external parameter characterising the stratification, e.g., the potential temperature increment across the PBL.
} 
profile taken after the Monin-Obukhov (1954) similarity theory and the velocity defect law. It had the following form:

$$
\ln \left(C_{g} \mathrm{Ro}\right)-A(\mu)=\left(\left(k / C_{g}\right)^{2}-B(\mu)\right)^{1 / 2},
$$$$
\sin \alpha=-k^{-1} B(\mu) C_{g} \operatorname{sign}(f)
$$

where $k$ is the von Karman constant, $\operatorname{Ro}=U_{g} /\left|f z_{0}\right|$ is the surface Rossby number, $A(\mu)$ and $B(\mu)$ are dimensionless coefficients to be determined empirically. At that time it was believed that $A$ and $B$ depend only on the Kazanski and Monin (1961) stratification parameter $\mu=\beta F_{\theta} /\left(|f| u_{*}^{2}\right)=u_{*} /|f L|$, where $F_{\theta}$ is the potential temperature flux at the surface, $\beta=g / T_{0}$ is the buoyancy parameter, $g$ is the acceleration due to gravity, $T_{0}=293 \mathrm{~K}$ is a reference value of the absolute temperature or the inverse thermal expansion coefficient, and $L=-u_{*}^{3} / \beta F_{\theta}$ is the Monin-Obukhov length scale. The use of the stratification parameter $\mu=u_{*}|f L|^{-1}$ in the resistance law results in the artificial self-correlation as both $\mu$ and $C_{g}$ depend on $u_{*}$. There were many attempts to determine $A(\mu)$ and $B(\mu)$ theoretically and/or from experimental data (see overview in Byun, 1991) but the data scatter was extremely large, which made the PBL resistance law nearly useless from the practical point of view.

Zilitinkevich and Esau $(2002,2005)$ advanced the theory and, using higher quality LES data, revealed that a great deal of the scatter was due to the incomplete set of governing parameters. They demonstrated that, besides the traditionally considered effect of the surface buoyancy flux $\beta F_{\theta}$, the static stability of the free flow above the PBL characterised by the Brunt-Vaisala frequency $N=\left(\beta \nabla_{z} \theta\right)^{1 / 2}$ also affects the PBL turbulence (mathematically - through the upper boundary condition for the potential temperature). In the new formulation of the resistance law functions, $A$ and $B$ became functions of composite stratification parameters:

$m_{A, B}=\left(\mu^{2}+C_{A, B} \mu_{N}^{2}\right)^{1 / 2}$

where $C_{A, B}$ are the empirical constants, and $\mu_{N}=\mathrm{N} /|f|$.

Certainly, the problem of completeness is equally relevant to direct numerical simulations (DNS) or field/lab experiments. Several authors have already noticed the effect of the imposed temperature stratification on $C_{g}$ and $\alpha$ (e.g. Byun, 1991). Using Arctic data, Overland and Davidson (1992) proposed empirical relationships:

$$
\begin{aligned}
C_{g} & =C_{g 0}-0.0069\left(N_{850} / 0.022\right)^{5} \quad \text { and } \\
\alpha & =14.7+9.6\left(N_{850} / 0.022\right)^{4}
\end{aligned}
$$

where $N_{850}$ is the Brunt-Vaisala frequency at $850 \mathrm{mb}$ surface (an empirically available proxy for the ambient stratification of the free atmosphere). Overland and Davidson found the coefficient $C_{g 0}=0.036$ in Eq. (3). The best fit to our LES data was found with $C_{g 0}=0.054$.

As follows from the above theory, the set of the governing dimensionless numbers $\left(\mu, \mu_{N}, \mathrm{Ro}\right)$ is complete. These numbers identify the steady-state, shear-driven, neutrally or stably stratified, barotropic PBL. However, the two problems remain. Firstly, the above dimensionless numbers are interdependent, that is they are not based on completely independent parameters: in particular, $f$ appears in all three numbers, and $u_{*}$ in the expression of $\mu$ obviously correlates with $U_{g}$ in the expression of Ro. Secondly, the theoretical resistance-law formulation is implicit: expresses $C_{g}=u_{*} / U_{g}$ through Ro and $m_{A}$, which includes $\mu$ and thus $u_{*}$.

In this study, we employ an alternative set of dimensionless numbers (based on the same set of the governing parameters) which allows determining $C_{g}$ and $\alpha$ explicitly. In Sect. 2 we introduce a modified set of the external governing parameters. In Sect. 3 we present the LES code and still unpublished details of the LES database DATABASE64, which provides empirical basis for our analysis; and in Sect. 4 we employ LES data to determine $C_{g}$ and $\alpha$ as functions of the external parameters.

\section{Parameter phase space}

We consider high-Reynolds-number, neutral or stable, steady-state, shear-driven barotropic PBLs over infinite, flat but aerodynamically rough surface developing against neutrally or stably stratified flow in a rotated frame of reference. In our case, the only source of the turbulent kinetic energy (TKE) is the shear of the mean flow driven by the horizontal pressure gradient $\nabla P$. The sinks of the TKE are its viscous dissipation (strongly enhances in the surface layer due to the surface friction) and its conversion to the potential energy most pronounced in the surface layer (where it is controlled by $\beta F_{\theta}$ ) and at the PBL upper boundary where it is affected by $N$ ). Employing the $\pi$-theorem (Buckingham, 1914), of the five governing parameters $\nabla P\left(\right.$ or $\left.U_{g}=f^{-1} \nabla(P / \rho)\right), z_{0}$, $f, \beta F_{\theta}$ and $N$ one could compose 3 independent dimensionless numbers:

$\operatorname{Ro}=U_{g} /\left(f z_{0}\right), \mu_{N}=N /|f|, \mu_{S}=\left(\beta F_{\theta} / f^{3} z_{0}\right)^{1 / 3}$.

Here, the surface-layer stability parameter $\mu_{S}$ does not include $u_{*}$, in contrast to the traditional parameter $\mu=\beta F_{\theta} /\left(|f| u_{*}^{2}\right)$. Physically, these numbers reflect the mentioned mechanisms of the TKE consumption.

The parameter phase space $\left(\operatorname{Ro}, \mu_{N}, \mu_{S}\right)$ include the following orthogonal subspaces: (Ro, 0,0$)$, the truly neutral PBL more or less easily reproduced in lab experiments and sometimes observed in the atmosphere or ocean (the simplest case from the theoretical point of view); (Ro, $\left.\mu_{N}, 0\right)$, the conventionally neutral PBL typically observed over the ocean in late summer and autumn; (Ro, $0, \mu_{S}$ ), nocturnal PBL typically observed during the night at low- and mid-latitudes. In the real atmosphere / ocean, all three dimensionless numbers are not-zero. The external stability parameters $\mu_{N}$ and $\mu_{S}$ are especially large at high latitudes, because of strong and persistent radiation imbalance (Overland and Guest, 1991) and long life-times of the stable PBLs, which eliminates the so-called residual layers separating the PBL from the free 
atmosphere at mid and low latitudes. Examples are given in Yague et al. (2001) for Antarctica and in Persson et al. (2002) for Arctic.

According to the above reasoning, we assume that $C_{g}$ and $\alpha$ are universal functions of Ro, $\mu_{N}$ and $\mu_{S}$. Implicitly, it implies that these parameters predominantly depend on largescale turbulence. Högström et al. (2002) gave observational evidences supporting this assumption. The role of large eddies in the momentum transport could be clearly seen over a water surface in a windy day as spots of ripples or "cat paws".

\section{Large-eddy simulation code and database}

LES data are attractive for this study as they could be easily obtained in controllable and properly idealized environment. The LES code LESNIC was developed at Uppsala University, Sweden, and the Nansen Environmental and Remote Sensing Centre, Norway. The LESNIC solves the momentum, temperature and continuity equations for incompressible Boussinesq fluid. The code employs a fully conservative 2 nd order central difference scheme for the skew-symmetric advection term, the 4th order Runge-Kutta scheme for time stepping, and a direct fractional-step pressure correction scheme for the continuity preservation. The computational mesh is staggered C-type mesh, which demands only prescribed turbulent fluxes as boundary conditions. The LESNIC employs the dynamic mixed closure. This closure parameterizes the sub-grid TKE dissipation with locally and instantly adjusting Smagorinsky closure to preserve some part of the inertial sub-range at the smallest resolved scales. The dynamic mixed closure excludes such undesirable tricks as manual tuning of sub-grid dissipation constants. This feature makes simulations robust over the whole practically required range of the external parameters. For detailed description of the LESNIC see Esau (2004), and for its intercomparisons see Fedorovich et al. (2004) and Beare et al. (2006).

Guermond et al. (2004) showed that the Smagorinsky closure complies with requirements of the Ladyzenskaya existence and uniqueness theorem. Hence, LESNIC provides numerical solution of regularized (filtered) equation of motions. The theorem implies that this solution converges to a solution of the Navier-Stokes equation. The convergence has been numerically tested for two cases of the isotropic homogeneous turbulence versus direct numerical simulations (Meyers et al., 2003) and, in the GABLS intercomparisons, for one case of stably stratified turbulence versus very high resolution LES (Beare et al., 2006). Both studies came to favourable conclusion that the relatively modest $64^{3}$ mesh is sufficient to keep simulation errors at the level less than 5\% of the total turbulent kinetic energy. We used these convergence estimates to guide computation of our LES database.
The LESNIC code was used to calculate a unique database (hereafter DATABASE64) of LES runs for the shear-driven PBL. Since DATABASE64 has not been described in details yet, we give below some information about it. DATABASE64 is built on the following principles applied to every run. The numerical mesh is an equidistant mesh with $64^{3}$ grid nodes. Figure 1 shows comparisons for some turbulence statistics resolved on $64^{3}$ and $128^{3}$ meshes. As expected, the largest difference is in the vertical component of the velocity fluctuation, $w^{\prime}$. Comparisons of the vertical momentum fluxes ${ }^{2} \tau=\left(\left(\overline{u^{\prime} w^{\prime}}\right)^{2}+\left(\overline{v^{\prime} w^{\prime}}\right)^{2}\right)^{1 / 2}$ reveal that the near surface damping of $w^{\prime}$ is almost compensated by amplification of the horizontal velocity fluctuations $u^{\prime}$ and $v^{\prime}$. So the difference in the vertical transport characteristics remains fairly small. This conclusion is also consistent with the Beare et al. (2006) LES intercomparisons study. The aspect ratio between the vertical and horizontal grid dimensions varies from 1:1 to $1: 4$ with the majority of data computed at 1:2 mesh. The physical resolution varies from run to run. It keeps approximately 30 to 45 vertical levels within the fully developed PBL. This resolution assures that more than 95\% of the TKE in the PBL core is explicitly resolved by the model (Meyers and Baelmans, 2004). One can estimate the resolved part of the TKE at the first computational level relative to the resolved TKE at $128^{3}$ mesh. Fluctuations at that level experience the strongest numerical damping. Our estimation suggests that about $80 \%$ of the TKE (and $65 \%$ of $\left.\overline{w^{\prime} w^{\prime}}\right)$ is still resolved at this level.

The PBL comprises only $1 / 2$ to $2 / 3$ of the depth of the computational domain. This prerequisite, along with the mesh anisotropy, assure that the largest eddies, up to those occupied the entire PBL depth, are not affected by the limited horizontal size of the LES domain. The lateral boundary conditions are periodic. The bottom boundary conditions are the followings: the turbulent flux of potential temperature at the surface $F_{\theta s}=\overline{\theta^{\prime} w^{\prime}}(z=0)$ is prescribed and therefore $F_{\theta s}$ is considered in this study as the external parameter; whereas the turbulent flux of momentum at the surface is computed instantly and pointwise using the log-law as $\sqrt{|\tau(x, y, 0, t)|}=u_{*}(x, y, 0, t)=\left|u_{1}\left(x, y, z_{1}, t\right)\right| / \kappa^{-1} \ln \left(z_{1} / z_{0}\right)$, where $u_{1}\left(x, y, z_{1}, t\right)$ is the computed flow velocity at the first computational level $z=z_{1}, z_{0}$ is the roughness length and $k=0.41$ is the von Karman constant. The above boundary conditions primarily affect the flow at $z_{1}$. The momentum flux, $\tau(x, y, z, t)$, at greater distances, $z$, from the surface primarily depends on resolved velocity fluctuations. Therefore the von Karman constant in the simulated log-layer in neutrally stratified PBLs defer from the value prescribed in the boundary conditions. Using the horizontally averaged values of the actual momentum flux and the velocity profile, it is possible to calculate $k$ in the LES as $k=\sqrt{\tau(z)} \ln \left(z / z_{0}\right) /|u(z)|$. The truly neutral

\footnotetext{
${ }^{2}$ Kinematic turbulent fluxes are considered since the LES is based on the incompressible Navier-Stokes equations.
} 

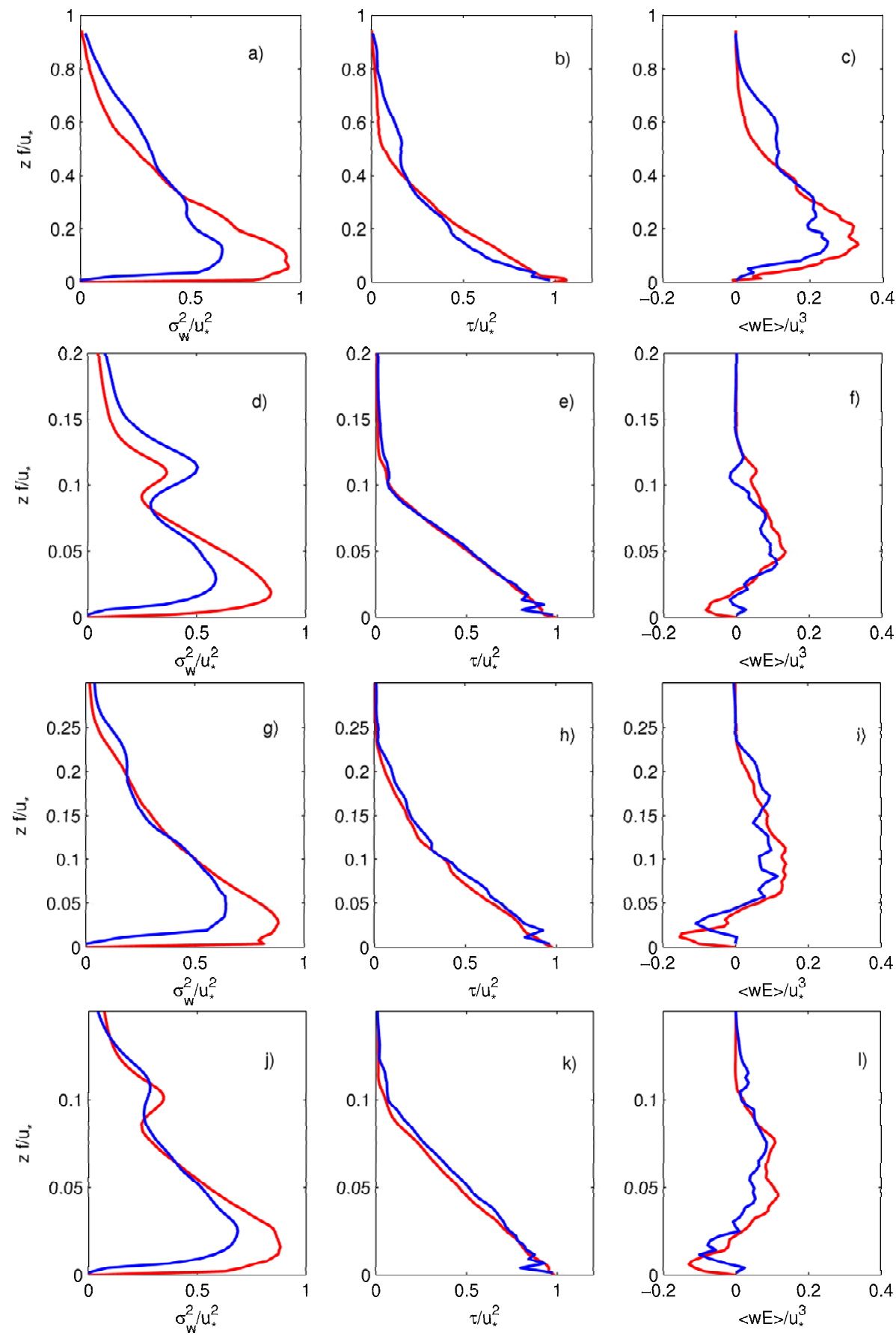

Fig. 1. Turbulence statistics from the truly neutral (a, b, c), conventionally neutral (d, e, f), nocturnal (g, h, i) and long-lived stably stratified $(\mathbf{j}, \mathbf{k}, \mathbf{l})$ LES rund at $64^{3}$ mesh (blue) versus the run at $128^{3}$ mesh (red). Comparison of normalized resolved statistics for the variance of the vertical velocity fluctuations ( $a, d, g, j)$, the vertical momentum transport (b, e, h, k) and the vertical transport of the TKE (c, f, i, l). All statistics are averaged over one hour after $14 \mathrm{~h}$ of the model spin-up.

runs in the DATABASE64 give $k=0.47$ within the resolved log-layer. This value is slightly larger than the commonly accepted range $0.37<k<0.43$ (e.g. Högström, 1985) in the atmospheric PBLs. However, it is in a good correspondence with laboratory experiments (e.g. Osterlund et al., 2000).
The initial conditions are: the laminar flow with the height-constant geostrophic velocity and given positive Brunt-Väisälä frequency. Thus, the simulated PBLs developed against the stably stratified atmosphere as it is typically observed. All LES run over 15 model hours. To test 
convergence of the turbulence statistics to the steady-state, a selected case $\left(\log _{10}(\mathrm{Ro})=5.67 ; \mu_{N}=300 ; \mu_{S}=10^{3}\right)$ run over $85 \mathrm{~h}$. Figure 2 shows that the steady state at the accuracy level 10\% has been reached within $14 \mathrm{~h}$.

The turbulent statistics are obtained through horizontal averaging of instant fluctuations (relative to the horizontally mean quantities) of the velocity and potential temperature at every node. In addition, the statistics are averaged over the last hour of simulations. Figure 3 shows a map of the DATABASE64 in the PBL parameter phase space. LES data correspond to the typical meteorological intervals such as observed during the SHEBA (Uttal et al., 2002; Persson et al., 2002) and the CASES-99 (Poulos et al., 2002) field campaigns. Recall that the majority of prior LES (see Zilitinkevich and Esau, 2002, 2003) provided data close to the following points in the parameter phase space: $\left(\mathrm{Ro}=10^{4}\right.$, $\left.\mu_{N}=0, \mu_{S}=0\right),\left(\operatorname{Ro}=10^{4}, \mu_{N}=180, \mu_{S}=0\right)$ and $\left(\mathrm{Ro}=10^{4}\right.$, $\mu_{N}=180, \mu_{S}=10^{3}$ ), which represented a serious obstacle for the development of analytical approximations.

\section{Universal dependences in different PBL regimes}

\subsection{Truly neutral PBL $($ Ro, 0,0$)$}

As already mentioned the traditional stable-PBL models did not account for the stratification of the free atmosphere. Therefore, in numerous publications, $C_{g}$ and $\alpha$ at $\mu=0$ were considered as functions of Ro only. Figure 4 shows this type of plots. They exhibit quite large scatter considered at the time as a "natural scatter" (see Csanady, 1974; Hess and Garratt, 2002). Our analysis of data from DATABASE64 leads to the conclusion that this scatter is largely attributed to the overlooked effect of $\mu_{N}$. Figure 5 shows the same dependencies but for selected data representing only the truly neutral PBLs. It demonstrates that in this regime $C_{g}$ and $\alpha$ can indeed be considered as universal functions of Ro.

In neutral stratification, Eq. (1) acquires the form

$$
\begin{aligned}
\frac{k}{C_{g}} \cos \alpha & =\ln C_{R}+\ln \mathrm{Ro}+\ln C_{g}-B, \\
\frac{k}{C_{g}} \sin \alpha & =-C_{R} A,
\end{aligned}
$$

where $C_{R}$ is the Rossby-Montgomery constant in the familiar expression $h_{E}=C_{R} u_{*} /|f|$ for the equilibrium neutral PBL depth, $h_{E}\left(C_{R}=0.65 \pm 0.05\right.$ after DATABASE64 and Coleman's (1999) direct numerical simulations). In this regime the dimensionless coefficients $A$ and $B$ become universal constants. Our analysis of data from DATABASE64 demonstrates their practical independence on Ro and thus confirms this conclusion. Rearranging the terms in Eq. (5) and neglecting small terms, such as $\ln C_{g}$, yields the following approximate version of this equation:

$C_{g}=C_{g 1}(\mathrm{Ro})=\frac{k}{\ln \operatorname{Ro}+C_{*}}$,

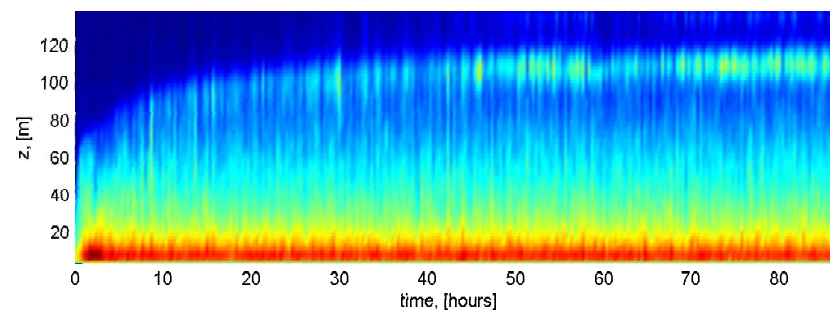

Fig. 2. Evolution of the TKE in the long LES run $\left(\log _{10}(\mathrm{Ro})=5.67, \mu_{N}=300, \mu_{S}=10^{3}\right)$. Dark blue colour shows no TKE in the flow, red colour shows the highest level of the TKE in the flow. The total depth-integrated TKE and the PBL depth at $14 \mathrm{~h}$ are within $10 \%$ of the mean TKE averaged over the time interval between 14 and $85 \mathrm{~h}$.

$$
\alpha=\arcsin \left(C_{\alpha} C_{g 1}(\mathrm{Ro})\right) \text {, }
$$

where $C_{*}=-4.2$ and $C_{\alpha}=4$ are matching constants determined from DATABASE64. Equation (6) becomes too rough at very small values of Ro, which, however are much beyond the geophysically interesting interval of Ro.

\subsection{Conventionally neutral PBL (Ro, $\left.\mu_{N}, 0\right)$}

The functions $A\left(\operatorname{Ro}, \mu_{N}\right)$ and $B\left(\right.$ Ro, $\left.\mu_{N}\right)$ exhibit strong dependence on $\mu_{N}$ (Zilitinkevich and Esau, 2005), which makes difficult their analytical approximation. However it is conceivable that at every fixed $\mu_{N}$ these functions should be similar to $A(\mathrm{Ro})$ and $B(\mathrm{Ro})$ given by Eqs. (5) or (6). Moreover, our analysis of data from DATABASE64 suggests that taking a fixed value of Ro, the resistance law coefficients $A$ and $B$ can be considered as self-similar functions of $\mu_{N}$. If so, the functions in question could be approximated as $C_{g}\left(\mathrm{Ro}, \mu_{N}\right)=C_{g 1}(\mathrm{Ro}) \cdot C_{g_{2}}\left(\mu_{N}\right)$ and $\alpha\left(\operatorname{Ro}, \mu_{N}\right)=\alpha_{1}(\mathrm{Ro}) \cdot \alpha_{2}\left(\mu_{N}\right)$. The first functions in these expressions are given by Eq. (6). We obtain the second functions from DATABASE64 by analysing the $\mu_{N^{-}}$ dependencies for every fixed Ro, which yields

$$
\begin{aligned}
C_{g} & =C_{g 1}(\mathrm{Ro}) \cdot\left(C_{N 1} \mu_{N}^{2 / 3}+1\right), \\
\alpha & =\sin ^{-1}\left(C_{\alpha} C_{g 1}(\mathrm{Ro}) \cdot\left(C_{N 2} \mu_{N}^{3 / 4}+1\right)\right),
\end{aligned}
$$

where $C_{N 1}=-5.8 \cdot 10^{-4}$ and $C_{N 2}=0.03$ are empirical constants determined with the accuracy $\pm 5 \%$. Figure 6 verifies Eq. (7) against DATABASE64.

Equations (7) showing quite strong dependence of $C_{g}$ and $\alpha$ on $\mu_{N}$ are based only on the DATABASE64. Certainly they need direct empirical validation. Recall that the PBL thickness, $h_{\mathrm{PBL}}$, also exhibit a strong dependence on $\mu_{N}$. Zilitinkevich and Esau $(2002,2003)$ have suggested and verified against the LES data the following dependence

$f h_{\mathrm{PBL}} / u_{*}=C_{R}\left(1+C_{N} \mu_{N}\right)^{-1 / 2}$,

where $C_{R}=0.65$ and $C_{N}=0.023$. In Fig. 7, we show that Eq. (8) correlates well with ERA-40 reanalysis, in which 


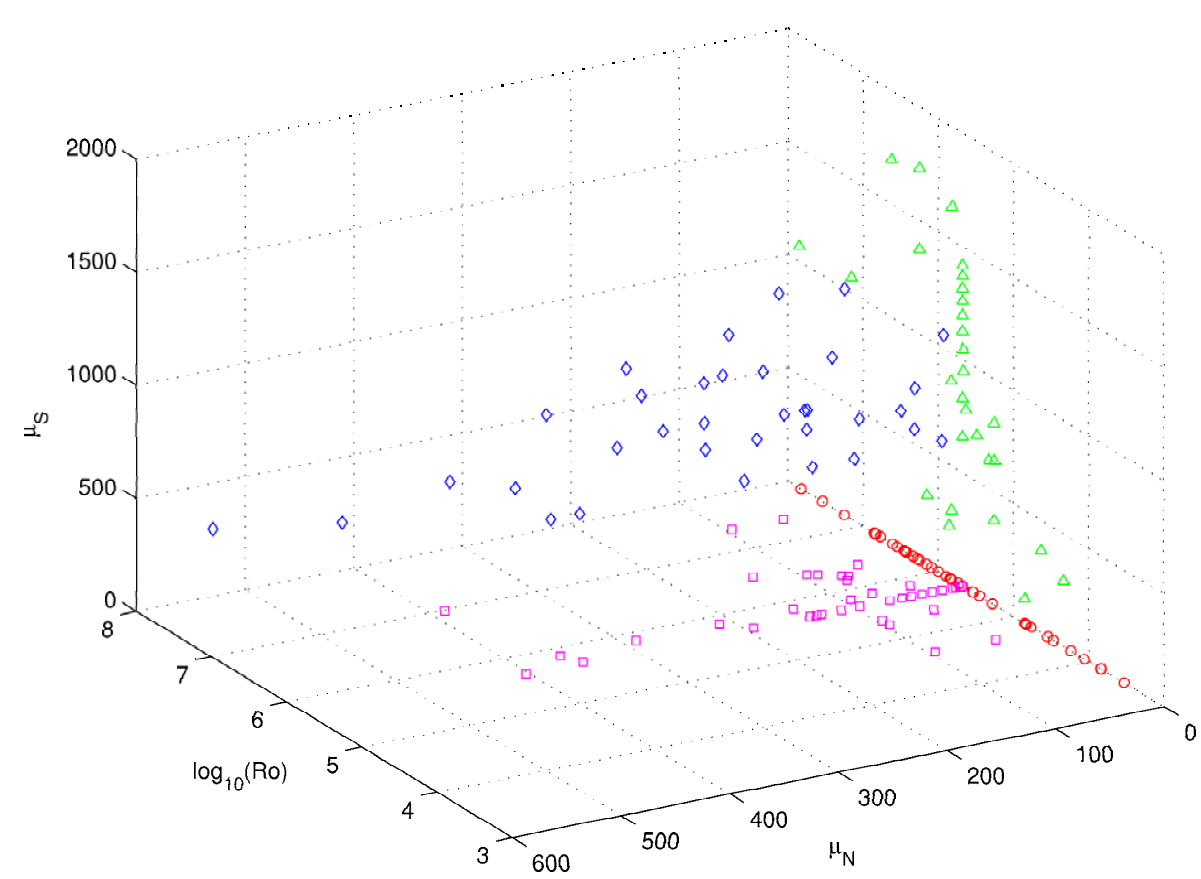

Fig. 3. A map of the DATABASE64 in the parameter space (Ro, $\left.\mu_{N}, \mu_{S}\right)$. Symbols: circles are truly neutral PBL (Ro,0, 0); squares are conventionally neutral PBL (Ro, $\left.\mu_{N}, 0\right)$; triangles are nocturnal PBL (Ro, $0, \mu_{S}$ ); diamonds are long-lived stably stratified PBL, that is the most general case $\left(\right.$ Ro, $\left.\mu_{N}, \mu_{S}\right)$. The PBL classification is after Zilitinkevich and Esau (2002).
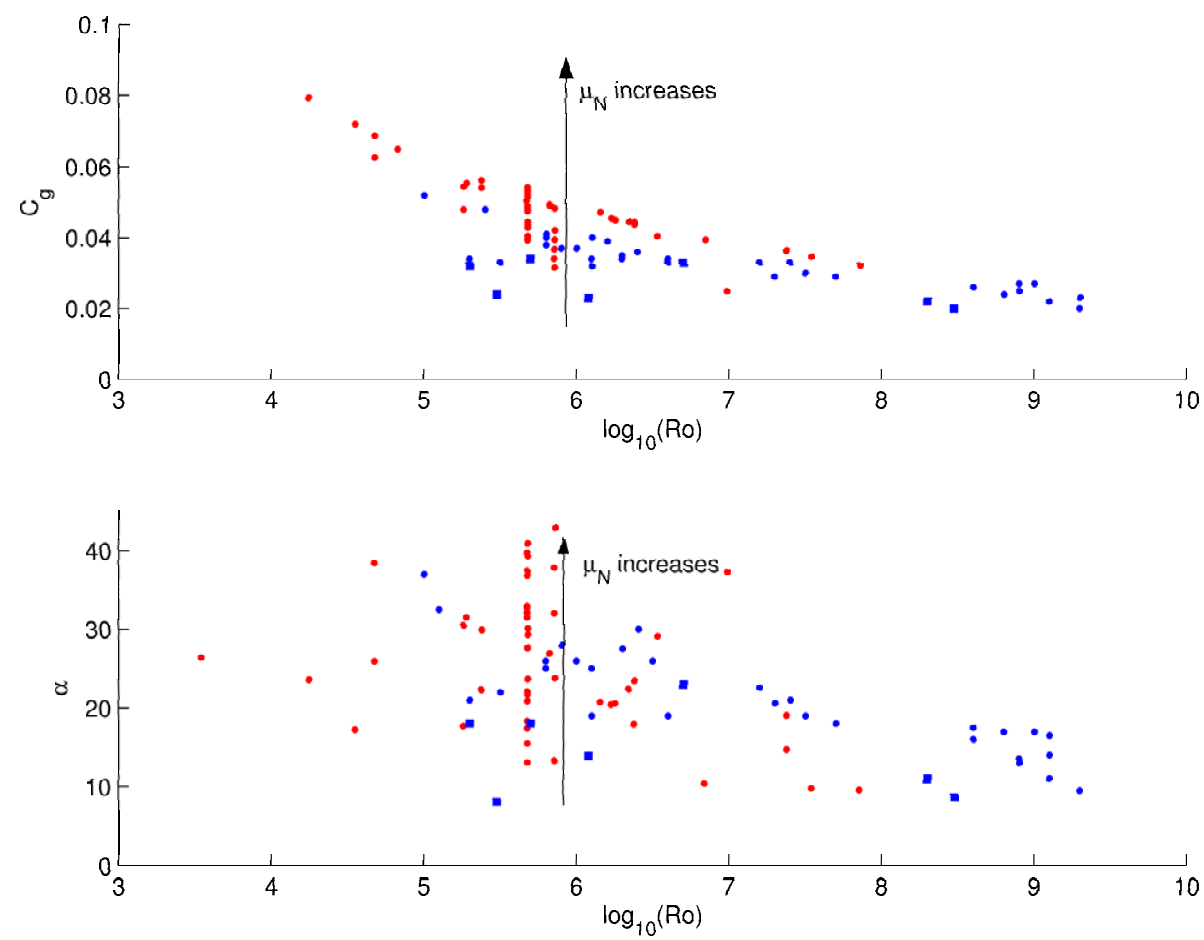

Fig. 4. Traditional presentation of $C_{g}$ and $\alpha$ in "near-neutral" PBLs as single-valued functions of Ro. Symbols: red circles are LES data from DATABASE64; blue circles are atmospheric data from Hess and Garratt (2002); blue squares are atmospheric data from Csanady (1974). Arrows show data scatter due to non-zero values of $\mu_{N}$. 

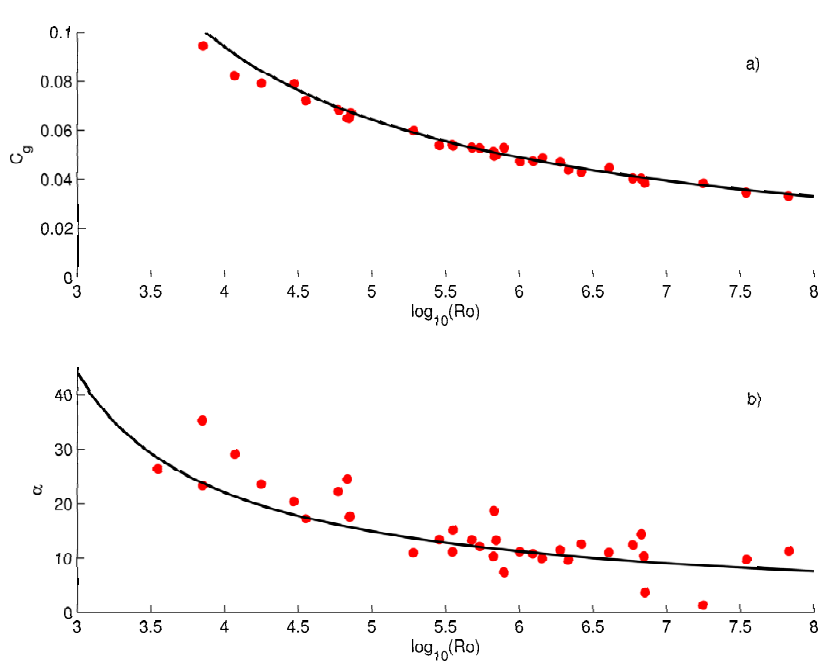

Fig. 5. $C_{g}$ and $\alpha$ in the truly neutral PBL shown as universal functions of Ro. Symbols are data form DATABASE64. Curves show analytical dependences after Eq. (6) with empirical constants $C_{*}=-4.2$ and $C_{\alpha}=4$.

$h_{\mathrm{PBL}}$ was calculated using the Troen and Mahrt (1986) bulkRichardson-number method. In turn, Miller et al. (1999) and Beljaars and Kohler (2000) compared the ERA-40 data and the LITE satellite data on $h_{\mathrm{PBL}}$. They reported a reasonable agreement in these two data sets.

\subsection{Nocturnal stably stratified PBL $\left(\operatorname{Ro}, 0, \mu_{S}\right)$}

In this regime the DATABASE64 also supports the parameter separation: $C_{g}\left(\mathrm{Ro}, \mu_{S}\right)=C_{g 1}(\mathrm{Ro}) \cdot C_{g 3}\left(\mu_{S}\right)$ and $\alpha\left(\operatorname{Ro}, \mu_{S}\right)=\alpha_{1}(\operatorname{Ro}) \cdot \alpha_{3}\left(\mu_{S}\right)$. Here, the first functions are given by Eq. (6), whereas the third functions are obtained from the DATABASE64 by analysing the $\mu_{S}$-dependences at every fixed Ro:

$$
\begin{aligned}
C_{g} & =C_{g 1}(\mathrm{Ro}) \cdot\left(\left(C_{S 1} \mu_{S}\right)^{3}+1\right), \\
\alpha & =\sin ^{-1}\left(C_{\alpha} C_{g 1}(\mathrm{Ro}) \cdot\left(\left(C_{S 2} \mu_{S}\right)^{3}+1\right)\right)
\end{aligned}
$$

where $C_{S 1}=-6.38 \cdot 10^{-4}$ and $C_{S 2}=0.0012$ are empirical constants determined with accuracy $\pm 5 \%$. Figure 8 verifies Eq. (9) against DATABASE64. It is worth mentioning that, in terms of Mahrt's (1998) classification, the DATABASE64 covers only weakly stable PBLs. All LES runs develop continues turbulence in time and space. However, the most stable runs with the largest $\mu_{S}$ exhibit significant variability of the second- and third-order turbulence statistics with periods from $30 \mathrm{~min}$ to $1 \mathrm{~h}$ - in remarkable agreement with CASES99 data (Banta et al., 2002; also L. Mahrt, personal communication).
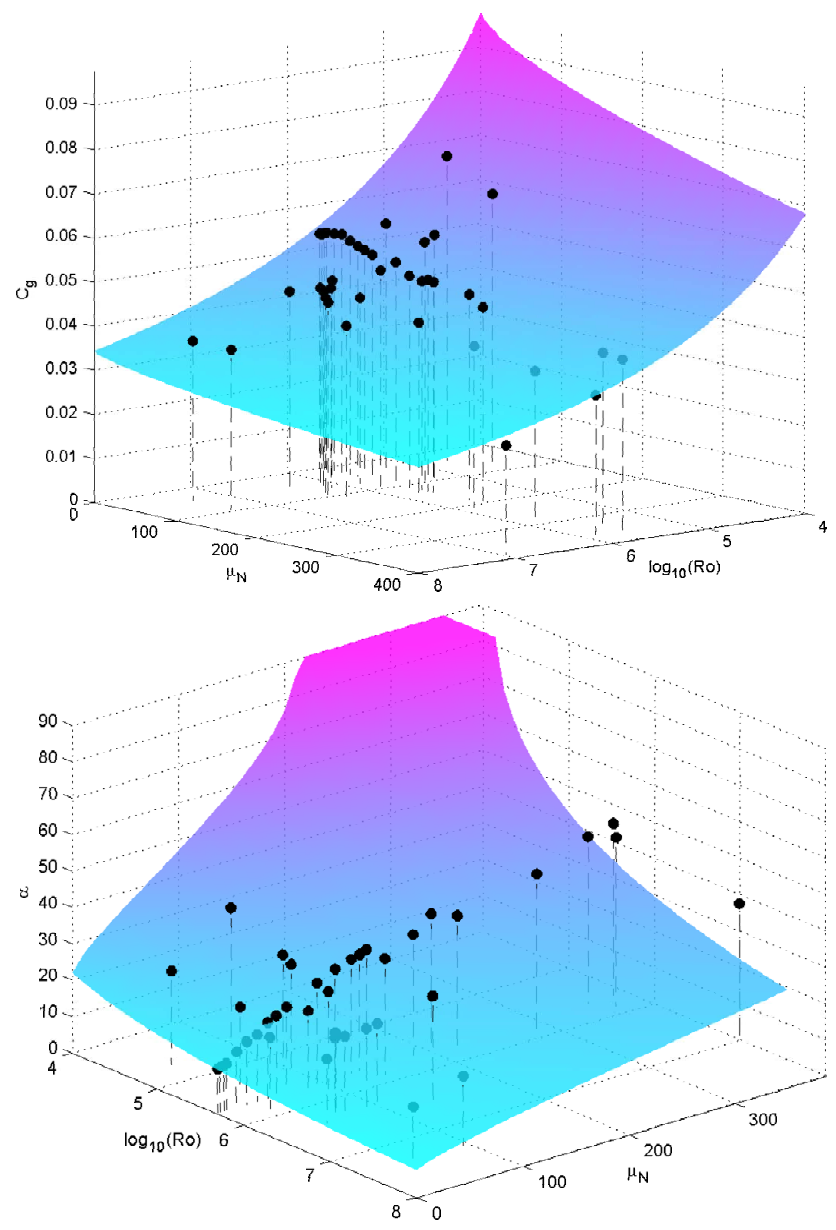

Fig. 6. $C_{g}$ and $\alpha$ in the conventionally neutral PBL shown as universal functions of Ro and $\mu_{N}$. Symbols are data form DATABASE64. Coloured iso-surfaces show analytical dependences after Eq. (7).

\subsection{Transition-regime exponents}

Equations (6), (7) and (9) can be used to calculate $C_{g}$ and $\alpha$ in weather-prediction, climate, wind-energy and airquality models. From the physical point of view it is interesting to consider the exponents in the $\mu_{N^{-}}$and $\mu_{S^{-}}$ dependences. As follows from Figs. 9 and 10 the exponents for $C_{g}$ are $(\ln \mathrm{Ro})^{-1}, \mu_{N}^{2 / 3}, \mu_{S}^{3}$; and the exponents for $\alpha$ are $(\ln \mathrm{Ro})^{-1}, \mu_{N}^{3 / 4}, \mu_{S}^{3}$. These empirically determined behaviour calls for theoretical explanation. However, it should be emphasised that the above approximations represent only the transition regime. At very large $\mu_{N}$ or $\mu_{S}$, and very small Ro, Eqs. (6) and (7) lose physical sense and give unreasonable values of $C_{g}<0$ and $\sin \alpha>1$. Thus, an asymptotic theory is needed to describe the whole range of the dimensionless numbers under consideration. Although the values of $\mu_{N}>350$ and $\mu_{S}>1500$ were never observed in the earth's atmosphere, they could be expected in the 


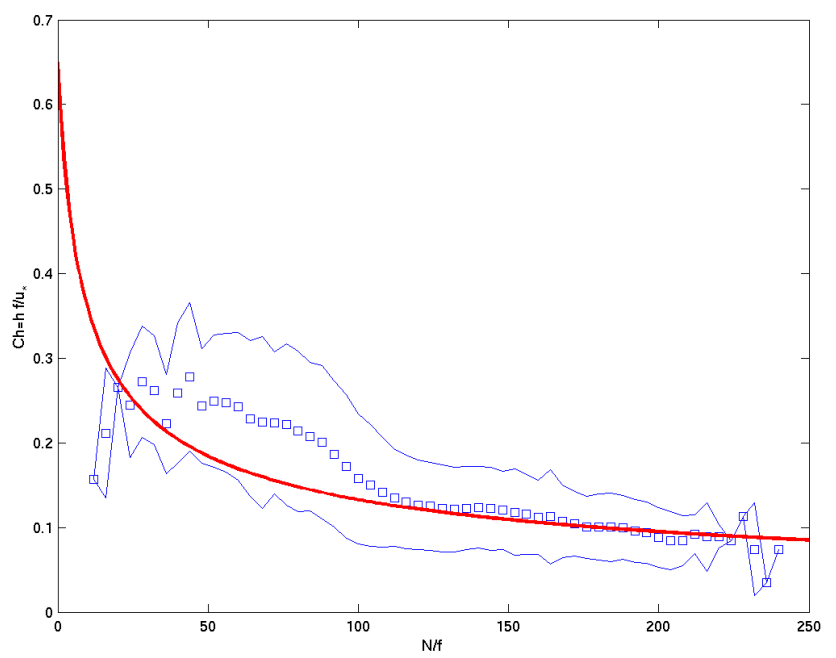

Fig. 7. The coefficient $C_{h}=h_{\mathrm{PBL}}|f| / u_{*}$ in the conventionally neutral case as a function of the imposed stratification parameter $\mu_{N}$. The read curve is an analytical dependence after Zilitinkevich and Esau (2003). Blue squares are the bin-averaged values derived from ERA-40 reanalysis data. The data sampled between 1958-2002 for January only. Blue curves denote one standard deviation interval of the data.

ocean. Moreover, Eqs. (6) and (7) fail at very large values of Ro quite possible at low latitudes. In this case Eq. (6) predicts unrealistically small $C_{g}$, which would contradict to empirical Dean's law (Dean, 1978). The Dean's law gives $\lim _{R o \rightarrow \infty} C_{g}=C_{f}^{1 / 2}=0.27 R e^{-1 / 8} \approx 0.03$ for $R e=10^{9}$ as typical in the PBL.

4.5 Extension of the model to the entire parameter phase space $\left(\operatorname{Ro}, \mu_{N}, \mu_{S}\right)$

Using the same parameter-separation method as in Sects. 4.1-4.3, we construct the following general expressions:

$$
\begin{aligned}
& C_{g}=\frac{\kappa}{\ln \mathrm{Ro}+C_{*}} \cdot\left(C_{N 1} \mu_{N}^{2 / 3}+1\right) \cdot\left(\left(C_{S 1} \mu_{S}\right)^{3}+1\right), \\
& \alpha=\arcsin \left(C_{\alpha} \frac{\kappa}{\ln \mathrm{Ro}+C_{*}} \cdot\left(C_{N 3} \mu_{N}^{3 / 4}+1\right) \cdot\left(\left(C_{S 3} \mu_{S}\right)^{3}+1\right)\right) .
\end{aligned}
$$

Figure 11 verifies Eq. (10) against DATABASE64 at fixed $\mathrm{Ro}=10^{5.67}=467740$, which corresponds to typical summertime atmospheric conditions $\left(U_{g}=5 \mathrm{~m} \mathrm{~s}^{-1}\right)$ at the latitude $45^{\circ} \mathrm{N}$ over agricultural landscape with $z_{0}=0.1 \mathrm{~m}$.

\section{Conclusions}

Numerous meteorological and oceanographic models require simple parameterization of the surface drag coefficient $C_{g}$ and the cross-isobaric angle $\alpha$ in terms of the resolved, largescale variables. Such relations have been studied extensively, but these efforts faced very large scatter in observational data.

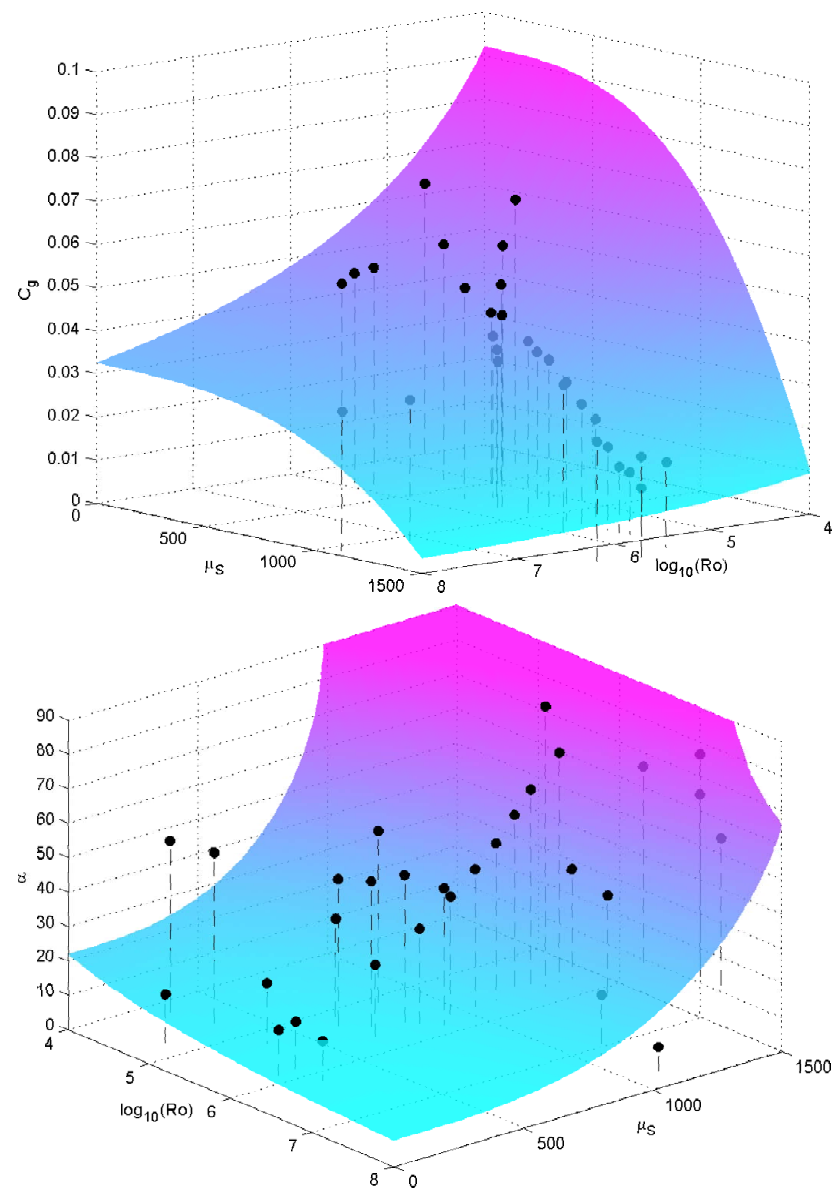

Fig. 8. $C_{g}$ and $\alpha$ in the nocturnal PBL shown as universal functions of Ro and $\mu_{S}$. Symbols are data form DATABASE64. Coloured iso-surfaces show analytical dependences after Eq. (9).

In the present paper, we consider this problem using simple similarity-theory reasoning and high-quality LES database DATABASE64, containing more than 130 LES runs, which cover more or less regularly the most practically interesting intervals of the parameter variability. We analyse the minimal complete set of parameters describing the boundary conditions and driving forces, and specify a plausible set of physically independent dimensionless numbers, namely the surface Rossby number Ro, the free-flow stability parameter $\mu_{N}$, and the surface-layer stability parameter $\mu_{S}$. Then, using DATABASE64, we empirically determine the exponents characterising the $\mu_{N^{-}}$and $\mu_{S}$-dependencies of $C_{g}$ and $\alpha$ in the transition regimes typical of the observed atmospheric PBLs. These exponents are $\left((\ln \mathrm{Ro})^{-1}, \mu_{N}^{2 / 3}, \mu_{S}^{3}\right)$ for $C_{g}$ and $\left((\ln \mathrm{Ro})^{-1}, \mu_{N}^{3 / 4}, \mu_{S}^{3}\right)$ for $\alpha$. Finally we employ linear matching to determine $C_{g}$ end $\alpha$ over the practically important region in the parameter phase space. The leastsquare accuracy of the proposed approximation is about $5 \%$ for $\mu_{N}<350, \mu_{S}<1500$ at the latitude 45 degrees. 

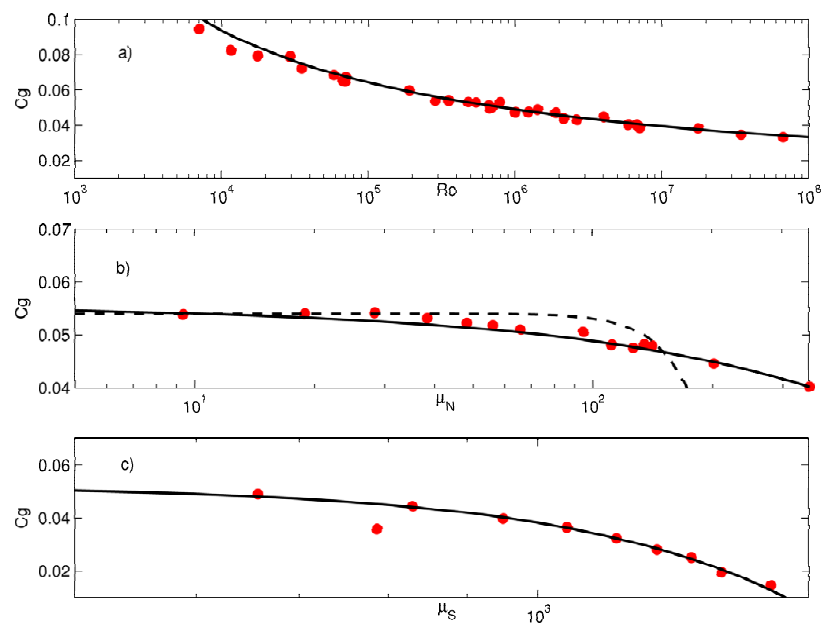

Fig. 9. Dependencies of $C_{g}$ on alternative dimensionless numbers: Ro (a), $\mu_{N}$ (b) and $\mu_{S}$ (c). Red dots are LES data from DATABASE64. Solid curves are proportional to $(\ln R o)^{-1}$ (a), $\mu_{N}^{2 / 3}$ (b) and $\mu_{S}^{3}$ (c). Dashed curve is an empirical fit suggested by Overland and Davidson (1992) in Eq. (3) with the LES corrected coefficient $C_{g 0}=0.054$.
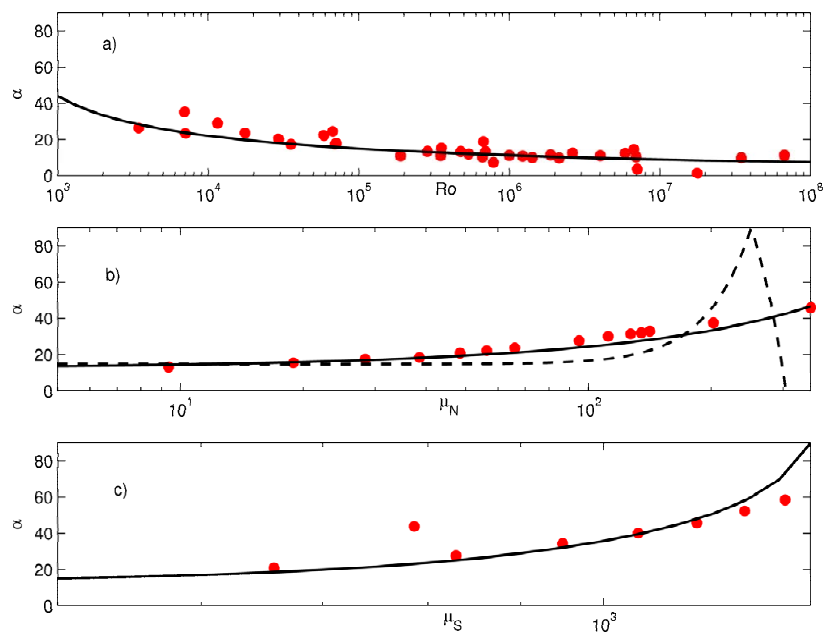

Fig. 10. Dependencies of $\alpha$ on alternative dimensionless numbers: Ro (a), $\mu_{N}$ (b) and $\mu_{S}$ (c). Red dots are LES data from DATABASE64. Solid curves are proportional to $(\ln R o)^{-1}$ (a), $\mu_{N}^{3 / 4}$ (b) and $\mu_{S}^{3}$ (c). Dashed curve is an empirical fit suggested by Overland and Davidson (1992) in Eq. (3).

Although this paper aims at practical applications, there are two major limitations for its immediate use. Firstly, we considered only transitional regimes covering limited interval of the parameter variability. Physically grounded approximations should include not only transitional but also asymptotic regimes corresponding to extreme values of the dimensionless numbers. Secondly, our conclusions are based on empirical post-processing of the LES data. That is the

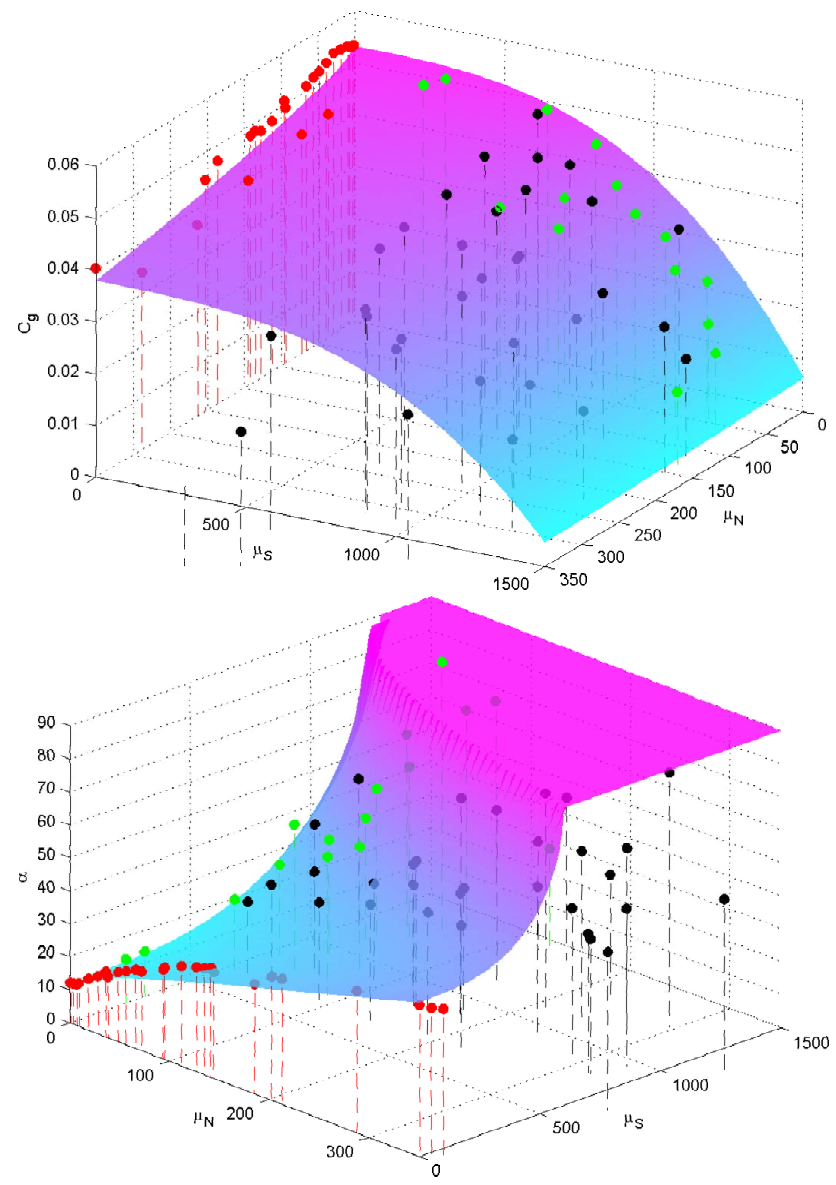

Fig. 11. $C_{g}$ and $\alpha$ in the PBL at fixed $\ln \mathrm{Ro}=5.67$ as universal functions of $\mu_{N}$ and $\mu_{S}$. Symbols are data form DATABASE64: red represent conventionally neutral PBL; green, nocturnal PBL; black, long-lived PBL. Coloured iso-surfaces show analytical dependences after Eq. (10). Dimmed symbols are below the proposed analytically determined universal iso-surface.

DATABASE64 is used not only to match the coefficients but also to determine the functional forms as such. Clearly, it is desirable to derive the functional forms theoretically. We plan to consider these problems in future work.

Acknowledgements. This work has been supported by the Norwegian project MACESIZ 155945/700, joint Norwegian-USA project ROLARC 151456/720, EU Marie Curie Chair Project MEXC-CT-2003-509742, ARO Project "Advanced parameterization and modelling of turbulent atmospheric boundary layers" contract number W911NF-05-1-0055; the NORDPLUS Neighbour 2005-2006 Project FI-51; and EU Project FUMAPEX EVK42001-00281.

Edited by: R. Grimshaw

Reviewed by: A. Petrosyan and three other referees 


\section{References}

Banta, R. M., Newsom, R. K., Lundquist, J. K., Pichugina, Y. L., Coulter, R. L., and Mahrt, L.: Nocturnal Low-Level Jet Characteristics Over Kansas During Cases-99, Boundary-Layer Meteorol., 105, 221-252, 2002.

Beare, R. J., MacVean, M. K., Holtslag, A. A. M., Cuxart, J. , Esau, I., Golaz, J.-C., Jimenez, M. A., Khairoutdinov, M., Kosovic, B., Lewellen, D., Lund, T. S., Lundquist, J. K., McCabe, A., Moene, A. F., Noh, Y., Raasch, S., and Sullivan, P.: An intercomparison of large-eddy simulations of the stable boundary layer, Boundary Layer Meteorol., in print, 2006.

Beljaars, A. and M. Kohler: LITE/ECMWF boundary layer height intercomparisons, http://www.ecmwf.int/research/physics/lite/, 2000, last visited 10 October 2005.

Buckingham, E.: On Physically Similar Systems: Illustrations of the Use of Dimensional Equations, Phys. Rev., 4, 345-376, 1914.

Byun, D.: Determination of similarity functions of the resistance laws for the planetary boundary layer using surface layer similarity functions, Boundary-Layer Meteorol., 57, 17-48, 1991.

Coleman, G. N.: Similarity statistics from a direct numerical simulation of the neutrally stratified planetary boundary layer, J. Atmos. Sci., 56(6), 891-900, 1999.

Csanady, G. T.: Equilibrium theory of the planetary boundary layer with an inversion lid, Boundary-Layer Meteorol., 6, 63-79, 1974.

Dean, R. B.: Reynolds number dependence of skin friction and other bulk flow variables in two dimensional rectangular duct flow, Trans. ASME: J. Fluids Eng., 100, 215-233, 1978.

Esau, I. N.: Simulation of Ekman boundary layers by large eddy model with dynamic mixed sub-filter closure, Environ. Fluid Mech., 4, 273-303, 2004.

Fedorovich, E., Conzemius, R., Esau, I., Chow, F. K., Lewellen, D., Moeng, C.-H., Pino, D., Sullivan, P., and Vila-Guerau de Arellano, J.: Entrainment into sheared convective boundary layers as predicted by different large eddy simulation codes, 16th AMS Symp. Boundary Layers and Turbulence, Portland, USA, 2004.

Guermond, J.-L., Oden, J. T., and Prudhomme, S.: Mathematical Perspectives on Large Eddy Simulation Models for Turbulent Flows, J. Math. Fluid Mech., 6, 194-248, 2004.

Hess, G. D. and Garratt, J. R.: Evaluating models of the neutral, barotropic planetary boundary layer using integral measures: Part I. Overview, Boundary-Layer Meteorol., 104, $333-$ $358,2002$.

Högström, U., Hunt, J. C. R., and Smedman, A.-S.: Theory and measurements for turbulence spectra and variances in the atmospheric neutral surface layer, Boundary-Layer Meteorol., 103, 101-124, 2002.

Högström, U.: Von Karman's Constant in Atmospheric Boundary Layer Flow: Re-evaluated, J. Atmos. Sci., 42, 263-270, 1985.

Kazanski, A. B. and Monin, A. S.: On turbulent regime above the surface layer, Izvestija AN SSSR Geophysical Series, 1, 165168,1961

Mahrt, L.: Stratified Atmospheric Boundary Layers and Breakdown of Models, Theoret. Comput. Fluid Dynamics, 11, 263279, 1998 .
Meyers, J., Geurts, B. J., and Baelmans, M.: Database analysis of errors in large-eddy simulation, Phys. Fluids, 15(9), 2740-2755, doi:10.1063/1.1597683, 2003.

Meyers, J. and Baelmans, M.: Determination of subfilter energy in large-eddy simulations, J. Turbulence, 5, 026, doi:10.1088/14685248/5/1/026, 2004.

Miller, S. D., Stephens, G. L., and Beljaars, A. C. M.: A Validation Survey of the ECMWF Prognostic Cloud Scheme using LITE, Geophys. Res. Letters, 26, 1417-1420, 1999.

Monin, A. S. and Obukhov, A. M.: Main characteristics of the turbulent mixing in the atmospheric surface layer, Trudy Geophys. Inst. AN. SSSR, 24(151), 153-187, 1954.

Osterlund, J. M., Johansson, A. V., Nagib, H. M., and Hites, M. H.: A note on the overlap region in turbulent boundary layers, Phys. Fluids, 12, 2159, 2000.

Overland, J. E. and Guest, P. S.: The Arctic Snow and Air Temperature Budget Over Sea Ice During Winter, J. Geophys. Res. 96(C3), 4651-4662, 1991.

Overland, J. E. and Davidson, K. L.: Geostrophic drag coefficients over sea ice, Tellus, 44A, 54-66, 1992.

Persson, O. P. G., Fairall, C. W., Andreas, E. L., Guest, P. S., and Perovich, D. K.: Measurements near the Atmospheric Surface Flux Group tower at SHEBA: Near-surface conditions and surface energy budget, J. Geophys. Res., 107, C10, 8045, doi:10.1029/2000JC000705, 2002.

Poulos, G., Poulos, G. S., Blumen, W., Fritts, D. C., Lundquist, J. K., Sun, J., Burns, S. P., Nappo, C., Banta, R., Newsom, R., Cuxart, J., Terradellas, E., Balsley, B. B., and Jensen, M. L.: CASES-99: A Comprehensive Investigation of the Stable Nocturnal Boundary Layer, Bull. Amer. Meteorol. Soc., 83(4), 555581, 2002.

Troen, I. and Mahrt, L.: A simple model of the atmospheric boundary layer: Sensitivity to surface evaporation, Boundary-Layer Meteorol., 37, 129-148, 1986.

Uttal, T., Curry, J. A., McPhee, M. G., Perovich, D. K., et al.: Surface Heat Budget of the Arctic Ocean, Bull. Amer. Meteorol Soc., 83, 255-276, 2002.

Yagüe, C., Maquedab, G., and Rees, J. M.: Characteristics of turbulence in the lower atmosphere at Halley IV station, Antarctica, Dynamics of Atmos. Oceans, 34, 205-223, 2001.

Zilitinkevich, S. S., Laikhtman, D. L., and Monin, A. S.: Dynamics of the boundary layer in the atmosphere, Izvestija, AN SSSR, FAO, 3(3), 297-333, 1967.

Zilitinkevich, S. S.: Velocity profiles, resistance law and the dissipation rate of mean flow kinetic energy in a neutrally and stably stratified planetary boundary layer, Boundary-Layer Meteorol., 46, 367-387, 1989.

Zilitinkevich, S. S. and Esau, I. N.: On integral measures of the neutral, barotropic planetary boundary layers, Boundary-Layer Meteorol., 104, 371-379, 2002.

Zilitinkevich, S. S. and Esau, I. N.: The effect of baroclinicity on the depth of neutral and stable planetary boundary layers, Quart. J. Roy. Meteorol. Soc., 129, 3339-3356, 2003.

Zilitinkevich, S. S. and Esau, I. N.: Resistance and Heat Transfer Laws for Stable and Neutral Planetary Boundary Layers: Old Theory, Advanced and Re-evaluated, Quart. J. Roy. Meteorol. Soc., 131, 1863-1892, 2005. 